\title{
Invasion speeds in microbial systems with toxin production and quorum sensing
}

${ }_{3}$ Sharon Bewick ${ }^{1 *}$, Phillip P.A. Staniczenko ${ }^{1,2 *}$, Bingtuan $\mathrm{Li}^{3}$, David Karig ${ }^{4} \&$ William F. Fagan ${ }^{1}$

$4{ }^{1}$ Department of Biology, University of Maryland, College Park, MD 20742, USA

${ }_{5}^{2}$ National Socio-Environmental Synthesis Center (SESYNC), Annapolis, MD 21401, USA

$6{ }^{3}$ Department of Mathematics, University of Louisville, Louisville, KY 40059, USA

$7 \quad{ }^{4}$ Johns Hopkins Applied Physics Laboratory, Fulton, MD 20723, USA

8 *Joint first-authors, correspondence should be addressed to sharon_bewick@ @otmail.com

The theory of invasions and invasion speeds has traditionally been studied in macroscopic systems. Surprisingly, microbial invasions have received less attention. Although microbes share many of the features associated with competition between larger-bodied organisms, they also exhibit distinctive behaviors that require new mathematical treatments to fully understand invasions in microbial systems. Most notable is the possibility for long-distance interactions, including competition between taxa mediated by diffusible toxins and cooperation among individuals of a single taxon using quorum sensing. In this paper, we model bacterial invasion using a system of coupled partial differential equations based on Fisher's equation. Our model considers a competitive system with diffusible toxins that, in some cases, are expressed in response to quorum sensing. First, we derive analytical approximations for invasion speeds in the limits of fast and slow toxin diffusion. We then test the validity of our analytical approximations and explore intermediate rates of toxin diffusion using numerical simulations. Interestingly, we find that toxins should diffuse quickly when used offensively, but that there are two optimal strategies when toxin is used as a defense mechanism. Specifically, toxins should diffuse quickly when their killing efficacy is high, but should diffuse slowly when their killing efficacy 
is low. Our approach permits an explicit investigation of the properties and characteristics of diffusible compounds used in non-local competition, and is relevant for microbial systems and select macroscopic taxa, such as plants and corals, that can interact through biochemicals.

\section{INTRODUCTION}

Understanding the factors governing the spread of a newly introduced species has long been an active area of research in the field of invasion biology $y^{1-4}$. Both empirical and theoretical studies have sought to clarify the organismal, community and environmental traits that can influence the invasion process $^{5-7}$. Early theoretical work, for example, highlighted the role of species fecundity and dispersal as major determinants of invasion success ${ }^{8}$. Later models were extended to include species properties such as stage structure ${ }^{9}$, Allee effects ${ }^{10}$, population-level heterogeneity ${ }^{11}$ and evolution ${ }^{12}$. Other studies examined the role of the environment ${ }^{13}$ and interspecific interactions ${ }^{14}$. Notably, negative interspecific interactions like competition were found to be particularly effective at slowing, stopping or even reversing the spread of an invading species ${ }^{15}$.

The concept of an established species or community acting as a barrier to invasion is not unique to macroscopic systems. Indeed, a similar idea recurs in the medical literature as 'colonization resistance,' where the invading taxon is a pathogen and the established community is the commensal microflora of a person's body ${ }^{16,17}$. In such cases, pathogen invasion speeds have a strong bearing on the outcome of a disease, and, with some fast-spreading infections, delayed intervention can have dire consequences ${ }^{18}$. It is well known that microscopic and macroscopic invasions have a number of features in common. For example, bacteria display many of the same competitive interactions as their macroscopic counterparts, including overuse of nutritional resources ${ }^{19,20}$ and exclusion from 
favorable locations in space ${ }^{21}$. However, despite the similarity between microscopic pathogens and macroscopic invasive species, few of the theoretical models from classical invasion biology have been translated and applied to study infections in humans or other organisms. Consequently, little is known about how the traits of different bacteria and bacterial communities affect the invasion speeds of pathogens.

The lack of theoretical work on bacterial invasion speeds is striking because, although similar in many ways to macroscopic systems, bacteria also demonstrate behaviors that are uncommon among macroscopic organisms and may affect the invasion process in distinctive ways. Most notable is bacterial production of small, diffusible compounds. Importantly, these compounds usually diffuse faster than the bacterial cells producing them, meaning that bacteria can strongly affect other bacteria—both competitors and collaborators—over relatively large distances. Therefore, unlike macroscopic competition, bacterial competition not only takes place through direct (cell-to-cell) contact, but also through indirect interactions mediated by toxin ${ }^{20}$ and frequently regulated through quorum sensing $(\mathrm{QS})^{22-26}$. Modeling these and other non-local effects is critical for understanding invasion in the microbial world. (It is worth noting that some plants and corals can produce diffusible biochemicals that influence the growth and survival of competitors ${ }^{27-30}$, a phenomenon known as allelopathy. As with bacterial invasions, the role of diffusible compounds on invasion speeds in these systems has also received little theoretical attention.)

Depending on the bacterial taxa involved, diffusible toxins can take a variety of different forms. In the simplest case, the toxin is a metabolic by-product. For example, the dominant member of the human skin microbiome, Propionibacterium acnes, produces propionic acid ${ }^{31}$ while the most common vaginal commensals, Lactobacillus spp., produce lactic acid ${ }^{32}$, both as by-products of fermen- 
tation. These two toxins acidify the environment, which, in turn, has been shown to inhibit a range of pathogens, including Staphylococcus aureus on the $\operatorname{skin}^{31}$ and Candida albicans, Gardnerella vaginalis and Mobiluncus spp. in the vagina ${ }^{33}$. Interestingly, fermentation by-products are not the only diffusible toxin produced by lactobacilli: members of this genus, along with some streptococci, also produce hydrogen peroxide $\left(\mathrm{H}_{2} \mathrm{O}_{2}\right)$, which is another small molecule that is effective against a range of catalase-negative human pathogens, including Staphylococcus aureus ${ }^{34}$ and the respiratory pathogen Haemophilus influenzae ${ }^{35}$.

In addition to acids and oxidizing agents, which typically act on a broad range of microbes, many bacteria also produce a range of more specialized antimicrobials. Of these, some of the best characterized are bacteriocins ${ }^{36}$, which are proteinaceous toxins that typically inhibit the growth of closely related bacterial strains (although occasionally bacteriocins have been observed suppressing more distantly related $\operatorname{taxa}^{37}$ ). Bacteriocins exhibit a high degree of variation among types. Class I bacteriocins (known as lantibiotics), for example, are small, thermostable peptides comprising only around 19-38 amino acid residues. By contrast, Class III bacteriocins are large, thermolabile proteins $(>30 \mathrm{kDa})$ with complex structures and functions ${ }^{38}$. The potencies of different bacteriocins are also highly heterogeneous, as are their modes of action ${ }^{39}$. The lantibiotic nisin, for example, binds to cell wall precursors, inhibiting cell wall formation in competitor taxa ${ }^{40}$. Similarly, lactic acid bacteria produce a range of bacteriocins that are capable of forming destructive pores in a competitor's cell membrane ${ }^{41}$. However, other bacteriocins, including colicins (particularly those produced by Escherichia coli), work by targeting protein synthesis in competitor taxa, negatively affecting both deoxyribonuclease $\mathrm{e}^{42}$ and ribonuclease ${ }^{43}$ activity. 
and well-being because we use natural antimicrobials in food ${ }^{37,44}$ and textile ${ }^{45}$ production, and, increasingly, as a source of novel pharmaceuticals ${ }^{46}$. Moreover, toxins produced by bacteria are a central component of our healthy microbiome, helping to protect us from pathogens that might otherwise colonize the surfaces of our bodies. One recent study, for example, identified approximately 4800 supposed bacteriocins from the Human Microbiome Project ${ }^{47}$. Despite the prevalence and importance of microbial toxins, few studies have specifically considered their effect on bacterial invasion and colonization. At a fundamental level, knowledge of how different types of antimicrobials affect colonization rate and success may offer insights into the evolutionary forces driving toxin acquisition and use. In terms of application, improved understanding of invasion speeds will allow us to identify unusually problematic pathogens, as well as ways of promoting bacteria that offer beneficial services, such as probiotics ${ }^{48}$.

Of the previous studies that have investigated the role of bacteriocins on invasion outcomes ${ }^{49-51}$, only a handful have examined trajectories of invasions ${ }^{52,53}$ and none have explicitly considered the role of toxin characteristics, for example size and mode/efficacy of killing. Strictly in terms of theoretical work, the majority of existing literature has focused on a few select topics: the role of bacteriocins in maintaining bacterial population coexistence ${ }^{49-51}$ and establishing microbial diversity ${ }^{54,55}$, cost-benefit analyses of bacteriocin production by bacteria ${ }^{56}$, and the environmental conditions favoring bacteriocin evolution ${ }^{57,58}$. The few studies that have examined invasion rates have done so either in the context of serial dilutions ${ }^{52}$ or with moving population boundaries ${ }^{53}$. However, neither of these approaches permits an examination of the properties of the toxins themselves, such as how physical characteristics like compound size or chemical structure-common determinants of toxin diffusion rate_-might affect pathogen invasion speeds. 
In this study, we use a system of coupled partial differential equations (PDEs) based on Fisher's equation to describe the invasion of a pathogen into a one-dimensional domain populated by an established competitor. We derive analytical approximations for the shape of the wavefront of a diffusible toxin under several limiting conditions. We then use these results to approximate pathogen invasion speeds under similar limiting conditions. We supplement our analytical treatment with numerical simulations, which allow us to explore additional scenarios that are not analytically tractable, including systems with intermediate rates of toxin diffusion and systems where toxin production is regulated by QS. In total, we consider four cases: i) the invading taxon constitutively produces a toxin; ii) the established taxon constitutively produces a toxin; iii) the invading taxon produces a toxin that is regulated by QS; and iv) the established taxon produces a toxin that is regulated by QS.

\section{METHODS}

For each system that we consider, we assume two bacterial taxa - the invader population, $A=$ $A(x, t)$, and the established population, $B=B(x, t)$. When the invader population produces a toxin, we denote the corresponding toxin profile $S=S(x, t)$. When the established population produces a toxin, we denote the toxin profile $T=T(x, t)$.

\section{Constitutive toxin production}

Eqn 1 describes a system in which two bacterial taxa both produce toxins: 


$$
\begin{aligned}
\frac{\partial A}{\partial t} & =D_{A} \frac{\partial^{2} A}{\partial x^{2}}+r_{A} A\left(1-A-\alpha_{A B} B\right)-k_{A T} A T \\
\frac{\partial S}{\partial t} & =D_{S} \frac{\partial^{2} S}{\partial x^{2}}+r_{S} A-m_{S} S \\
\frac{\partial B}{\partial t} & =D_{B} \frac{\partial^{2} B}{\partial x^{2}}+r_{B} B\left(1-B-\alpha_{B A} A\right)-k_{B S} B S \\
\frac{\partial T}{\partial t} & =D_{T} \frac{\partial^{2} T}{\partial x^{2}}+r_{T} B-m_{T} T
\end{aligned}
$$

where $D_{A}$ and $D_{B}$ are diffusion coefficients for invader and established bacterial taxa respectively and $D_{S}$ and $D_{T}$ are diffusion coefficients for their toxins. Bacterial growth rates are given by $r_{A}$ and $r_{B}$, toxin production rates by $r_{S}$ and $r_{T}$ and toxin decay rates by $m_{S}$ and $m_{T}$. We assume that local competition between the invader and the established taxon follows Lotka-Volterra dynamics ${ }^{50}$ with interaction terms $\alpha_{A B}$ and $\alpha_{B A}$, representing, for example, competition for space or nutrients in the immediate vicinity of competing cells. The toxin produced by each bacterial taxon acts at a distance and kills competitor cells at rates $k_{B S}$ and $k_{A T}$. Linear determinacy conditions for this PDE system-which will be important for interpreting our numerical results—are included in Appendix I.

For simplicity, we restrict our analysis to systems with $D_{A}=D_{B}$ and $r_{A}=r_{B}$ and focus on two cases: only the invading taxon produces a toxin and only the established taxon produces a toxin. When only the invading taxon produces a toxin, Eqn 1 reduces to

$$
\begin{aligned}
\frac{\partial A}{\partial t} & =D_{A} \frac{\partial^{2} A}{\partial x^{2}}+r_{A} A\left(1-A-\alpha_{A B} B\right) \\
\frac{\partial S}{\partial t} & =D_{S} \frac{\partial^{2} S}{\partial x^{2}}+r_{S} A-m_{S} S \\
\frac{\partial B}{\partial t} & =D_{B} \frac{\partial^{2} B}{\partial x^{2}}+r_{B} B\left(1-B-\alpha_{B A} A\right)-k_{B S} B S
\end{aligned}
$$


and when only the established taxon produces a toxin, Eqn 1 reduces to

$$
\begin{aligned}
\frac{\partial A}{\partial t} & =D_{A} \frac{\partial^{2} A}{\partial x^{2}}+r_{A} A\left(1-A-\alpha_{A B} B\right)-k_{A T} A T \\
\frac{\partial B}{\partial t} & =D_{B} \frac{\partial^{2} B}{\partial x^{2}}+r_{B} B\left(1-B-\alpha_{B A} A\right) \\
\frac{\partial T}{\partial t} & =D_{T} \frac{\partial^{2} T}{\partial x^{2}}+r_{T} B-m_{T} T
\end{aligned}
$$

\section{QS-regulated toxin production}

$$
\begin{aligned}
\frac{\partial A}{\partial t} & =D_{A} \frac{\partial^{2} A}{\partial x^{2}}+r_{A} A\left(1-A-\alpha_{A B} B\right)-k_{A T} A T \\
\frac{\partial Q}{\partial t} & =D_{Q} \frac{\partial^{2} Q}{\partial x^{2}}+v_{0} A+v_{\max } A \frac{Q^{n_{Q}}}{Q^{n_{Q}}+K_{Q}^{n_{Q}}}-m_{Q} Q \\
\frac{\partial S}{\partial t} & =D_{S} \frac{\partial^{2} S}{\partial x^{2}}+\psi\left(v_{0} A+v_{\max } A \frac{Q^{n_{Q}}}{Q^{n_{Q}}+K_{Q}^{n_{Q}}}\right)-m_{S} S \\
\frac{\partial B}{\partial t} & =D_{B} \frac{\partial^{2} B}{\partial x^{2}}+r_{B} B\left(1-B-\alpha_{B A} A\right)-k_{B S} B S \\
\frac{\partial R}{\partial t} & =D_{R} \frac{\partial^{2} R}{\partial x^{2}}+w_{0} B+w_{\max } B \frac{R^{n_{R}}}{R^{n_{R}}+K_{R}^{n_{R}}}-m_{R} R \\
\frac{\partial T}{\partial t} & =D_{T} \frac{\partial^{2} T}{\partial x^{2}}+\eta\left(w_{0} B+w_{\max } B \frac{R^{n_{R}}}{R^{n_{R}}+K_{R}^{n_{R}}}\right)-m_{T} T
\end{aligned}
$$

where $Q=Q(x, t)$ and $R=R(x, t)$ are densities of QS singaling chemical produced by the invading pathogen and established taxon, respectively, $D_{Q}$ and $D_{R}$ are chemical diffusion coefficients 
for the two QS signals and $m_{Q}$ and $m_{R}$ are QS-signal decay rates. We assume that singaling chemicals and toxins are both produced at a 'leakage' rate of $v_{0}$ and a maximum 'on' rate of $v_{\max }$ by the invading taxon and, correspondingly, at rates $w_{0}$ and $w_{\max }$ by the established taxon. We also assume density-dependent production of the QS signals, which we describe by a Hill function where $K_{Q}$ and $K_{R}$ are the threshold densities for half-maximal signal production and $n_{Q}$ and $n_{R}$ determine the change in signal production around the threshold densities. By using the same threshold densities, 'leakage' and 'on' rates for the singaling molecules and the toxins that they regulate, we are essentially assuming that both diffusible compounds are regulated by the same promoter. This is the simplest scenario, although systems with differential regulation are also possible. To capture differences in expression levels between singaling chemicals and toxins, we scale toxin production by factors $\psi$ and $\eta$ for invading and established taxa, respectively.

As with our analysis of constitutive toxin production, we focus on two cases: only the invading taxon produces a toxin and only the established taxon produces a toxin. When only the invading taxon produces a toxin, Eqn 4 reduces to

$$
\begin{aligned}
& \frac{\partial A}{\partial t}=D_{A} \frac{\partial^{2} A}{\partial x^{2}}+r_{A} A\left(1-A-\alpha_{A B} B\right) \\
& \frac{\partial Q}{\partial t}=D_{Q} \frac{\partial^{2} Q}{\partial x^{2}}+v_{0} A+v_{\max } A \frac{Q^{n_{Q}}}{Q^{n_{Q}}+K_{Q}^{n_{Q}}}-m_{Q} Q \\
& \frac{\partial S}{\partial t}=D_{S} \frac{\partial^{2} S}{\partial x^{2}}+\psi\left(v_{0} A+v_{\max } A \frac{Q^{n_{Q}}}{Q^{n_{Q}}+K_{Q}^{n_{Q}}}\right)-m_{S} S \\
& \frac{\partial B}{\partial t}=D_{B} \frac{\partial^{2} B}{\partial x^{2}}+r_{B} B\left(1-B-\alpha_{B A} A\right)-k_{B S} B S
\end{aligned}
$$

and when only the established taxon produces a toxin, Eqn 4 reduces to 


$$
\begin{aligned}
& \frac{\partial A}{\partial t}=D_{A} \frac{\partial^{2} A}{\partial x^{2}}+r_{A} A\left(1-A-\alpha_{A B} B\right)-k_{A T} A T \\
& \frac{\partial B}{\partial t}=D_{B} \frac{\partial^{2} B}{\partial x^{2}}+r_{B} B\left(1-B-\alpha_{B A} A\right) \\
& \frac{\partial R}{\partial t}=D_{R} \frac{\partial^{2} R}{\partial x^{2}}+w_{0} B+w_{\max } B \frac{R^{n_{R}}}{R^{n_{R}}+K_{R}^{n_{R}}}-m_{R} R \\
& \frac{\partial T}{\partial t}=D_{T} \frac{\partial^{2} T}{\partial x^{2}}+\eta\left(w_{0} B+w_{\max } B \frac{R^{n_{R}}}{R^{n_{R}}+K_{R}^{n_{R}}}\right)-m_{T} T
\end{aligned}
$$

As with toxins, most singaling chemicals are small molecules and therefore usually diffuse faster than the cells producing them, so we assume $D_{Q}, D_{R} \geq D_{A}, D_{B}$.

\section{Numerical solutions}

Although invasion speeds are defined, mathematically, on an infinite domain, we confirm analytical results and obtain additional results using numerical solutions of Eqns 2, 3, 5 and 6 on large but finite domains. Simulations begin with the established taxon at a constant population density (equal to its single-taxon equilibrium) across the entire, finite domain. We then introduce a small pulse of the invading taxon at the center of the domain. After allowing for a period of transient dynamics, we record the position of the invader wavefront at fixed time intervals to determine the invasion speed, which is constant. For all simulations where the invader produces a toxin, we set the domain to be large enough so that invader and toxin densities remain below a small threshold level $\left(1 \times 10^{-4}\right.$ molecules per unit length) at domain boundaries. Software was written in Python 2.7.10 and uses a finite-difference method for solving PDEs. 
174

175

\section{Constitutive toxin production}

\section{Analytical approximations for toxin wavefronts}

We derive expressions for toxin wavefronts with the following assumptions: toxins are not inhibitory $\left(k_{B S}=0\right.$ and $\left.k_{A T}=0\right)$, the invading taxon is not affected by local competition $\left(\alpha_{A B}=0\right)$, the established taxon is strongly inhibited by local competition $\left(\alpha_{B A}=2\right)$ and the growth and diffusion rates of the two bacterial taxa are identical $\left(r_{A}=r_{B}=r_{C}\right.$ and $\left.D_{A}=D_{B}=D_{C}\right)$.

With these assumptions, the wavefront of the invading taxon can be approximated as the solution to Fisher's equation and the wavefront of the established taxon as one minus the expression for the invader wavefront:

$$
\begin{aligned}
& A(x, t) \approx \frac{1}{1+e^{\frac{x}{2 D_{C}}-r_{C} t}} \\
& B(x, t) \approx 1-\frac{1}{1+e^{\frac{x}{D_{C}}-r_{C} t}}
\end{aligned}
$$

From Eqn 7, we can derive approximations for toxin wavefronts (see Appendix II). When the invader produces the toxin, the toxin wavefront is given by

$$
\begin{array}{ll}
S(x, t) \approx-\frac{r_{S}}{2 m_{S}}\left(e^{\sqrt{\frac{m_{S}}{D_{S}}}\left(x-2 D_{C} r_{C} t\right)}-2\right) & \text { for } x<2 D_{C} r_{C} t \\
S(x, t) \approx \frac{r_{S}}{2 m_{S}} e^{-\sqrt{\frac{m_{S}}{D_{S}}}\left(x-2 D_{C} r_{C} t\right)} & \text { for } x>2 D_{C} r_{C} t
\end{array}
$$


When the established taxon produces the toxin, the toxin wavefront is given by

$$
\begin{array}{ll}
T(x, t) \approx \frac{r_{T}}{2 m_{T}} e^{-\sqrt{\frac{m_{T}}{D_{T}}}\left(x-2 D_{C} r_{C} t\right)} & \text { for } x<2 D_{C} r_{C} t \\
T(x, t) \approx-\frac{r_{T}}{2 m_{T}}\left(e^{\sqrt{\frac{m_{T}}{D_{T}}}\left(x-2 D_{C} r_{C} t\right)}-2\right) & \text { for } x>2 D_{C} r_{C} t
\end{array}
$$

187

When the invader produces the toxin, the two limiting invasion speeds are

$$
\begin{aligned}
c_{0}^{*} & =2 \sqrt{D_{A} r_{A}\left(1-\alpha_{A B}\right)} \\
c_{\infty}^{*} & =2 \sqrt{D_{A} r_{A}\left(1-\alpha_{A B}\left(1-\frac{k_{B S} r_{S}}{2 m_{S} r_{B}}\right)\right)} \\
c_{\infty}^{*} & =2 \sqrt{D_{A} r_{A}}
\end{aligned}
$$

for $k_{B S} r_{S}<2 m_{S} r_{B}$

for $k_{B S} r_{S}>2 m_{S} r_{B}$

When the established taxon produces the toxin, the two limiting invasion speeds are 


$$
\begin{aligned}
c_{0}^{*} & =2 \sqrt{D_{A} r_{A}\left(1-\alpha_{A B}-\frac{k_{A T} r_{T}}{r_{A} m_{T}}\right)} \\
c_{\infty}^{*} & =2 \sqrt{D_{A} r_{A}\left(1-\alpha_{A B}-\frac{k_{A T} r_{T}}{2 r_{A} m_{T}}\right)}
\end{aligned}
$$

Notice that in both Eqns 10 and 11 invasion speeds are faster when the toxin diffuses quickly compared to when it diffuses slowly. This is an intuitive result. However, as we shall see below, this outcome is reversed in some scenarios where linear determinacy no longer holds.

We use numerical simulations to test the validity of our analytical approximations in Eqns 10 and 11 , and to explore intermediate toxin diffusion rates. Because we are interested in how toxin killing efficacy $\left(k_{B S}, k_{A T}\right)$ and diffusion rate $\left(D_{S}, D_{T}\right)$ interact to affect invasion speeds, we set the remaining parameters in Eqns 2 and 3 to $D_{A}=D_{B}=1, r_{A}=r_{B}=r_{S}=r_{T}=1, m_{S}=m_{T}=1$, $\alpha_{A B}=0.5$ and $\alpha_{B A}=5$.

\section{Numerical solutions when the invading taxon produces a toxin}

As expected, a non-diffusing (purely local) toxin produced by the invader does not affect its invasion speeds. In this case, the invader wavefront moves at speeds (Eqn 10a) consistent with classic results ${ }^{14}$ from invasion ecology (Fig. 1). However, invasion speeds increase and approach the analytical approximation for infinitely-fast toxin diffusion (Eqns 10b and 10c) as diffusion rates increase from $D_{S}=1$ to $D_{S}=100,000$. Notice that in the limit $D_{S} \rightarrow \infty$ for $k_{B S} r_{S} \geq 2 m_{S} r_{B}$ toxin killing is so rapid that the established taxon is completely eliminated and the invader moves unimpeded at its maximum (Fisher's) invasion speed. Therefore, depending on the particular combination of toxin killing efficacy and diffusion rate, the invasion speed of a pathogen that produces a toxin can range 


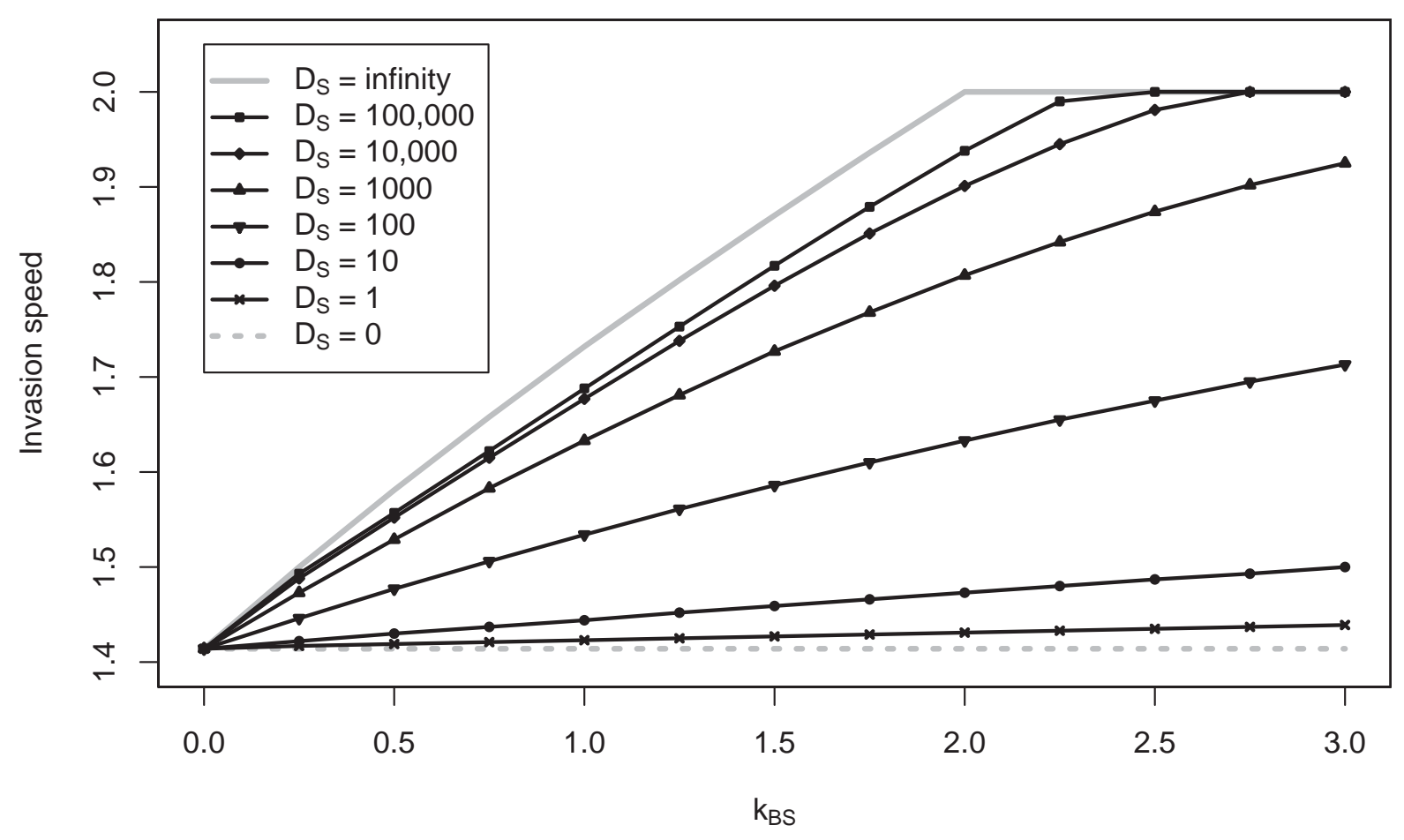

Figure 1: Invasion speeds when the invading taxon produces a toxin. Markers represent individual simulation runs and no markers indicate theoretical results (in this and subsequent figures). Invasion speeds increase from the classic theoretical result with no toxin (grey curve labeled as $D_{S}=0$, Eqn 10a) and gradually approach the theoretical approximation for the limit of fast toxin diffusion (grey curve labeled as $D_{S}=$ infinity, Eqn 10b) as diffusion rates increase from $D_{S}=1$ to $D_{S}=$ 100,000 . In the limit of fast toxin diffusion for $k_{B S} \geq 2$ the established taxon has been completely eliminated and the invader moves unimpeded at its maximum speed (labeled as $D_{S}=$ infinity, Eqn 10c), which for the set of parameters used is at $c_{\infty}^{*}=2$. 
from a lower bound given by classic results on invasion in a competitive system ${ }^{14}$ to an upper bound given by Fisher's spreading speed ${ }^{59}$.

\section{Numerical solutions when the established taxon produces a toxin}

Invasion speeds are expected to decrease when toxin is used as a defense mechanism by the established taxon. In keeping with this expectation, we find that invasion speeds decrease with increasing toxin killing efficacy until the invader is not able to colonize at all when $k_{A T}$ is high (Fig. 2). Interestingly, however, invasion speeds do not appear to agree well with our approximation for the limit of slow toxin diffusion (Eqn 11a). This is because the linear determinacy conditions required for this approximation only hold at very small $k_{A T}$ when $\alpha_{A B}$ is large (see the final condition, Eqn 16d, in Appendix I). We see much better agreement with the slow-diffusion approximation for simulations with the same parameter values except with $\alpha_{A B}=0.05$ instead of $\alpha_{A B}=0.5$ (Fig. S1). Linear determinacy is satisfied much more easily when $D_{T}$ is large (see Eqns $15 \mathrm{c}$ and 16d), and invasion speeds gradually approach our approximation for fast toxin diffusion (Eqn 11b) as diffusion rates increase from $D_{T}=1$ to $D_{T}=100,000$.

One interesting consequence of the failure of linear determinacy at low toxin diffusion rates involves the relationship between killing efficacy and $D_{T}$. We find that invasion speeds decrease with increasing killing efficacy at a decreasing rate for small $D_{T}$ (concave up), but at an increasing rate for large $D_{T}$ (concave down, notice the increase in downward curvature with increasing $D_{T}$ in the left-hand panel of Fig. 2). As a result, when toxin killing efficacy is low, a slowly diffusing toxin results in the largest reduction in invasion speed; however, when toxin killing efficacy is high, a quickly diffusing toxin results in the largest reduction in invasion speed. This suggests two strategies 

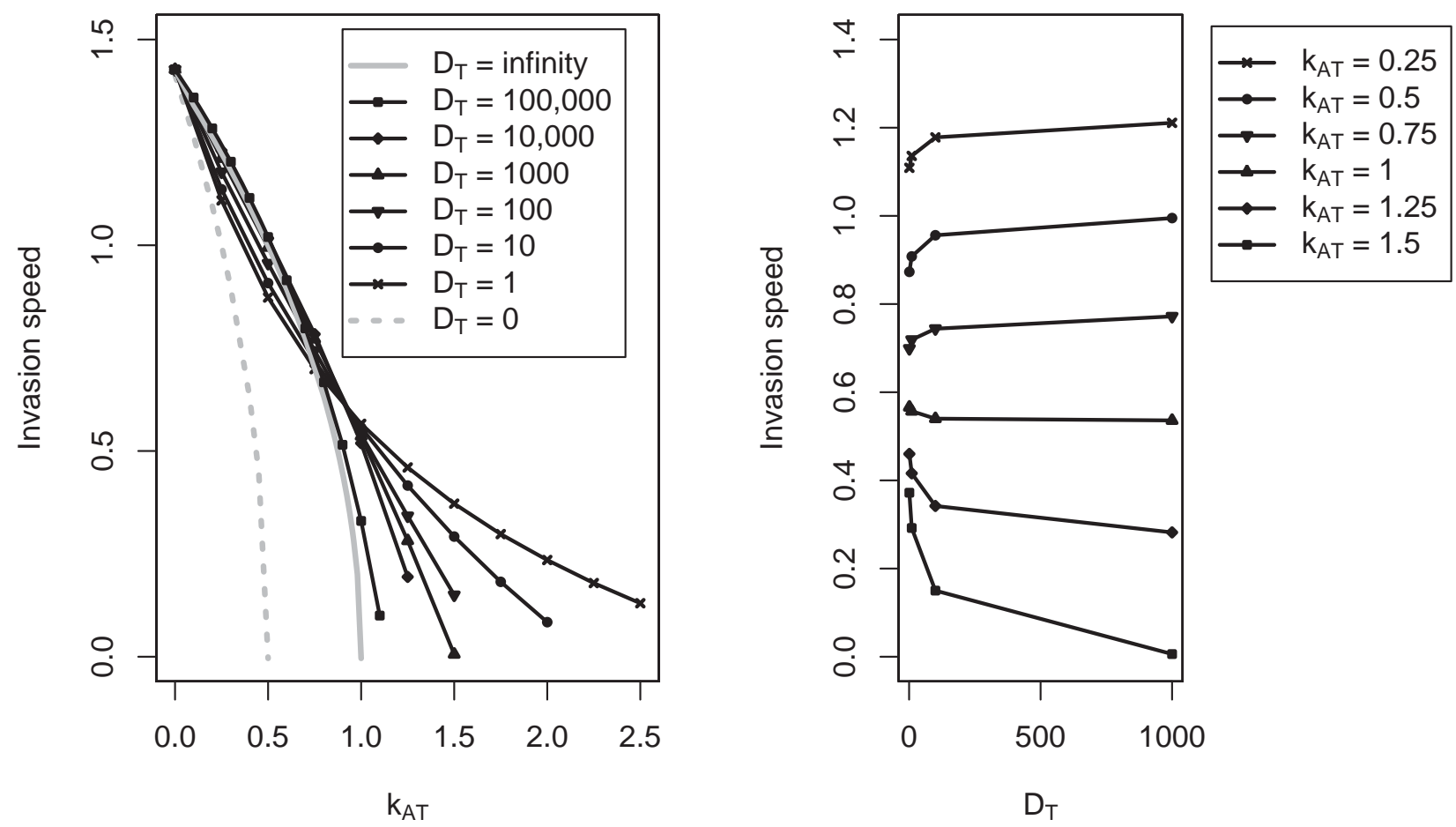

Figure 2: Invasion speeds when the established taxon produces a toxin. Left: Invasion speeds do not agree well with the theoretical approximation for the limit of slow toxin diffusion (grey curve labeled as $D_{T}=0$, Eqn 11a) because the linear determinacy conditions required for this approximation only hold at very small $k_{A T}$ (see main text). However, invasion speeds gradually approach the theoretical approximation for the limit of fast toxin diffusion (grey curve labeled as $D_{T}=$ infinity, Eqn 11b) as diffusion rates increase from $D_{T}=1$ to $D_{T}=100,000$. Interestingly, invasion speeds decrease at a decreasing rate at small $D_{T}$ but decrease at an increasing rate at large $D_{T}$ (notice the increase in downward curvature with increasing $D_{T}$ ). The value of $k_{A T}$ at which the invader cannot colonize decreases with increasing $D_{T}$; failure to colonize is associated with negative invasion speeds, which are omitted for clarity. Right: The change in profile of invasion-speeds curves as $D_{T}$ increases suggests two optimal strategies for toxin production: i) the toxin should not diffuse too quickly when $k_{A T}$ is small, e.g., when $k_{A T}=0.5$, invasion speeds are slower at low $D_{T}$; and ii) the toxin should diffuse more quickly when $k_{A T}$ is large, e.g., when $k_{A T}=1.5$, invasion speeds are slower at high $D_{T}$. 
for toxin production by an established taxon: i) the toxin should not diffuse too quickly (small $D_{T}$ ) when $k_{A T}$ is small; and ii) the toxin should diffuse more quickly (large $D_{T}$ ) when $k_{A T}$ is large. These two strategies can be clearly identified in the right-hand panel of Fig. 2.

\section{QS-regulated toxin production}

Compared to constitutive toxin production, QS will generally reduce the amount of toxin in the system. Consequently, invasion speeds are expected to decrease when QS regulates toxin production by the invader, but increase when QS regulates toxin production by the established taxon. However, as QS signals are often metabolically less costly than toxins, QS may serve to prevent unnecessary toxin production when bacterial cell density is insufficient for toxin to have a significant effect on the invasion process. Indeed, empirical studies have shown that toxin production often takes place under QS regulation ${ }^{22,23}$.

\section{Unfortunately, the complexity of the equations involving QS-regulated toxin production means} that, unlike with constitutive toxin production, we are not able to derive analytical expressions for invasion speeds. As such, we focus on numerical solutions to Eqns 5 and 6. Similar to the previous section, we are interested in how toxin killing efficacy $\left(k_{B S}, k_{A T}\right)$, toxin diffusion rate $\left(D_{S}, D_{T}\right)$ and, now, signaling chemical diffusion rate $\left(D_{Q}, D_{R}\right)$ interact to affect invasion speeds. For most parameters, we assume the same values as were used previously. However, when the invader produces toxin, we set the local competitive effects of the invader to $\alpha_{B A}=0.5$. This balances competition due to local and non-local effects, allowing us to focus closer examination on the impact of QS regulation. In addition, we set $m_{Q}=m_{R}=1, \psi=1, \eta=0.5, v_{0}=w_{0}=v_{\max }=w_{\max }=0.5, K_{Q}=K_{R}=0.5$ and $n_{Q}=n_{R}=100$ in Eqns 5 and 6. Notice that, with these parameter choices, $\eta=0.5$ implies 
that total toxin production by the established population is half as high in the scenario with QS regulated toxin production as compared to the scenario with constitutive toxin production. We make this assumption because it allows us to investigate the effect of QS regulated toxin production over a larger range of killing efficacies

\section{Numerical solutions when the invading taxon produces a toxin regulated by $Q S$}

To aid interpretation of results for systems with toxin production by the invader, we consider invasion speeds scaled between two extreme scenarios: no toxin production (slowest invasion speeds, which we set to $c^{\prime}=0$ ) and toxin production without QS (fastest invasion speeds, which we set to $c^{\prime}=1$ ). From Eqn 5, it is clear that there are two intermediate states between these two extremes: i) a lowspeed state where there is only leakage of the toxin, i.e., the system is below the QS-threshold; and ii) a high-speed state where additional toxin production has been turned 'on', i.e., the system is above the QS-threshold. Depending on system parameters and initial conditions, some systems will naturally transition into and remain in a low-speed state, whereas others will transition into and remain in a high-speed state. For example, when toxin killing efficacy, $k_{B S}$, is small, the density of the established taxon remains large enough to suppress the density of the invader through direct competition to such an extent that the invader cannot produce enough singaling chemical at the wavefront to exceed the QS-threshold. As a result the system is more likely to transition into a low-speed rather than high-speed state.

At killing efficacies of $k_{B S}=1$ and $k_{B S}=2$, systems naturally transition into high-speed states, even from initial conditions with no toxin or QS signal present (i.e., when only a small population of invader cells are introduced). For these systems, QS initially suppresses invasion 

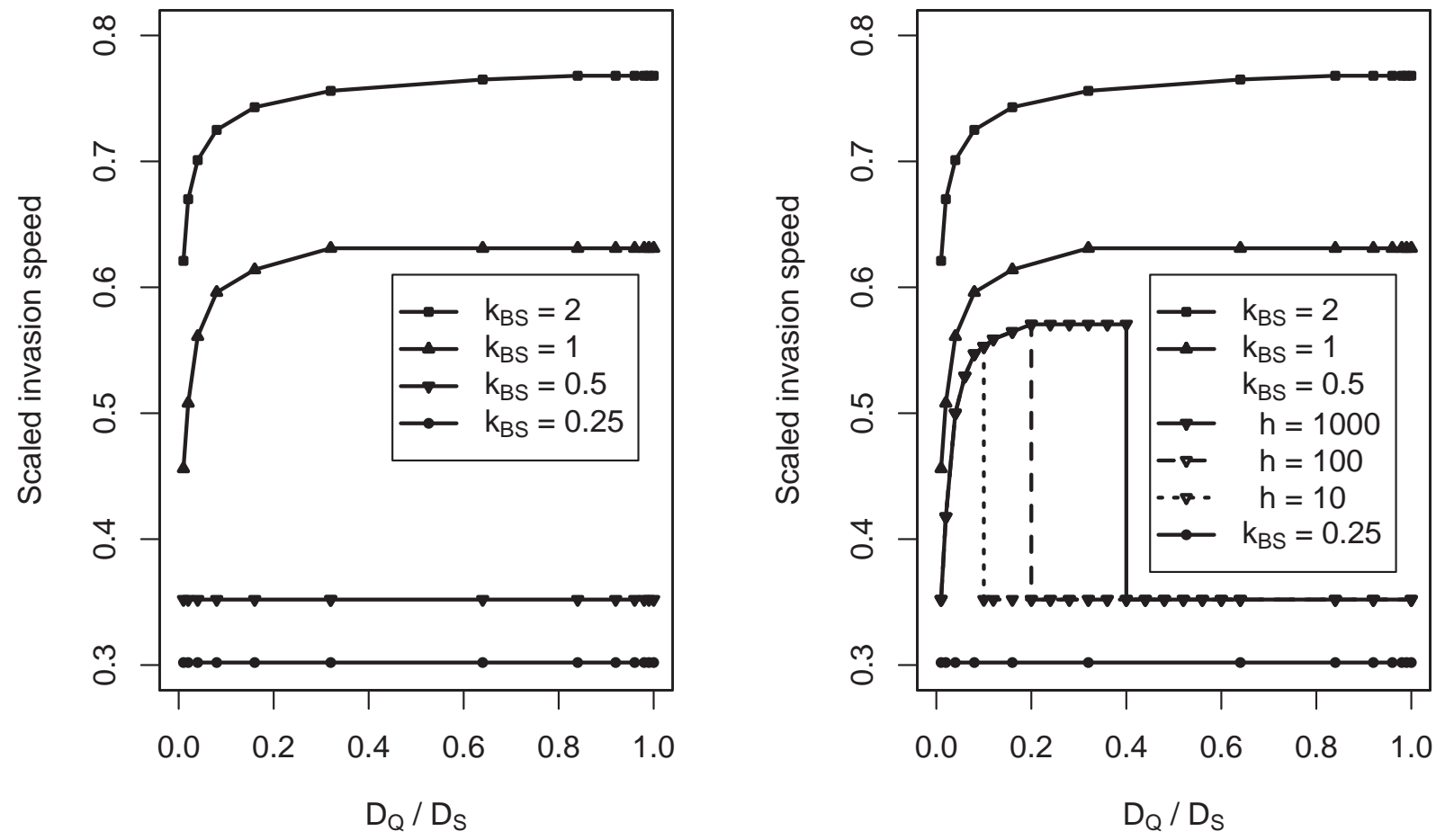

Figure 3: Scaled invasion speeds when the invading taxon produces a toxin regulated by quorum sensing, without (left) and with (right) the application of a spike in singaling chemical at the invader wavefront. Invasion speeds are scaled between no toxin (slowest invasion speeds, $c^{\prime}=0$ ) and toxin production without quorum sensing (fastest invasion speeds, $c^{\prime}=1$ ). Results are shown for $D_{S}=100$ and invasion speeds do not change significantly for $D_{Q}>D_{S}$. Left: Systems with $k_{B S}=0.25$ and $k_{B S}=0.5$ naturally transition into low-speed states, which are independent of $D_{Q}$. By contrast, systems with $k_{B S}=1$ and $k_{B S}=2$ naturally transition into high-speed states and quorum sensing suppresses invasion speeds when $D_{Q}$ is relatively small. However, as $t \rightarrow \infty$ the toxin will eventually diffuse from behind the invader wavefront and result in a constant invasion speed that is independent of $D_{Q}$. Smaller values of $D_{Q}$ require longer times to reach constant invasion speeds, which, given that simulations run for a finite amount of time, explains the monotonically increasing curves for $k_{B S}=1$ and $k_{B S}=2$. Right: The effect of applying a fixedamount spike in singaling chemical with height $h=1000$. Spiking has no effect on systems that naturally transition into high-speed states. However, with $k_{B S}=0.5$, the spike drives the system into a high-speed state at small $D_{Q}$, but dissipates at large $D_{Q}$ before sufficient singaling chemical has accumulated to shift states and the system remains in a low-speed state. Applying smaller amounts of singaling chemical ( $h=10$ and $h=100)$ reduces the value of $D_{Q}$ at which the system can be forced into a high-speed state. For the system with $k_{B S}=0.25$, even a spike with $h=1000$ is insufficient to force the system into a high-speed state. 
speeds, particularly when $D_{Q}$ is small (Fig. 3, left-hand panel). This is because QS restricts toxin production, with smaller $D_{Q}$ resulting in lower concentrations of toxin at the invader wavefront and therefore slower invasion speeds. However, as $t \rightarrow \infty$ toxin eventually diffuses from behind the invader wavefront. Because this accumulation of toxin is large compared to any differences in toxin loss due to different $D_{Q}$, invasion speeds essentially become independent of $D_{Q}$ over long time periods. Not unexpectedly, smaller values of $D_{Q}$ require longer times to reach limiting invasion speeds. This explains why curves for $k_{B S}=1$ and $k_{B S}=2$ increase monotonically in Fig. 3, which were all run for the same number of simulated time steps.

In contrast to the systems with $k_{B S}=1$ and $k_{B S}=2$, a comparable system with $k_{B S}=0.5$ is bistable: for the same initial conditions, the system with $k_{B S}=0.5$ naturally transitions into a low-speed state, but can be forced into a high-speed state by applying a fixed spike of QS signal at the invader wavefront. Successful transition into the high speed state depends on the amount of QS signal applied and its diffusion rate (Fig. 3, right-hand panel). For a small QS-spike with small $D_{Q}$, the spike drives the system into a high-speed state, where it remains indefinitely. However, for a small QS-spike with large $D_{Q}$, the spike dissipates before sufficient singaling chemical has accumulated, causing the system to transition back to a low-speed state, where it remains indefinitely. Thus, all else being equal, larger QS spikes are required to force systems with larger $D_{Q}$ into a highspeed state. For the comparable system with $k_{B S}=0.25$, no amount of QS-spike we tried was enough to force the system into a high-speed state.

The observed relationships between scaled invasion speeds and $D_{Q} / D_{S}$ (shown for $D_{A}=$ $\left.D_{B}=1\right)$ are similar for $D_{A}=D_{B}=10$ and $D_{S}=1000$, and $D_{A}=D_{B}=100$ and $D_{S}=10,000$, i.e., when $D_{S} / D_{A}=100$. The relationships are present but less pronounced when $D_{S} / D_{A}=10$ 
and $D_{S} / D_{A}=1000$. Patterns are qualitatively similar when 'leakage' and 'on' rates are set to more biologically-realistic values of $v_{0}=0.05$ and $v_{\max }=0.95$ instead of $v_{0}=v_{\max }=0.5$, although the reduction in basal amounts of toxin due to the lower 'leakage' rate means that systems are less likely to naturally transition into high-speed states (Fig. S2).

\section{Numerical solutions when the established taxon produces a toxin regulated by QS}

As suggested above, QS regulation over toxin production by the established taxon is expected to increase invasion speeds. However, because the initial density of the established taxon across the finite domain is so much larger than that of the invader, singaling chemical and toxin are quickly produced at their maximum rates, meaning that QS regulation (and therefore $D_{R}$ ) has a minimal effect on invasion speeds. Consequently, when the total toxin production is the same under QS regulated and constitutive toxin production, i.e., $\eta=1$, there is essentially no difference in the relationship between invasion speed, $D_{T}$, and $k_{A T}$, regardless of whether or not QS occurs (not shown). When we consider QS regulation using a lower toxin expression level $(\eta=0.5)$, curves for invasion speed versus toxin killing efficacy are, unsurprisingly, less pronounced. That is, curves are flatter and closer to their maximum value, which corresponds to no defensive toxin production. As a result, changes in curvatures for the different toxin diffusion rates are less dramatic. Nevertheless, the general profiles of the curves are similar with and without QS (Fig. 4). This means that the same qualitative patterns seen with constitutive toxin production (Fig. 2) are also present when toxin production is regulated by QS — at low $k_{A T}$ invasion speeds are slowest when $D_{T}$ is low (e.g., $\left.D_{T}=10\right)$ but at high $k_{A T}$ invasion speeds are slowest when $D_{T}$ is high (e.g., $\left.D_{T}=1000\right)$. Invasion speeds are very similar when 'leakage' and 'on' rates are set to more biologically-realistic values of $w_{0}=0.05$ and $w_{\max }=0.95$ instead of $w_{0}=w_{\max }=0.5($ Fig. S3). 


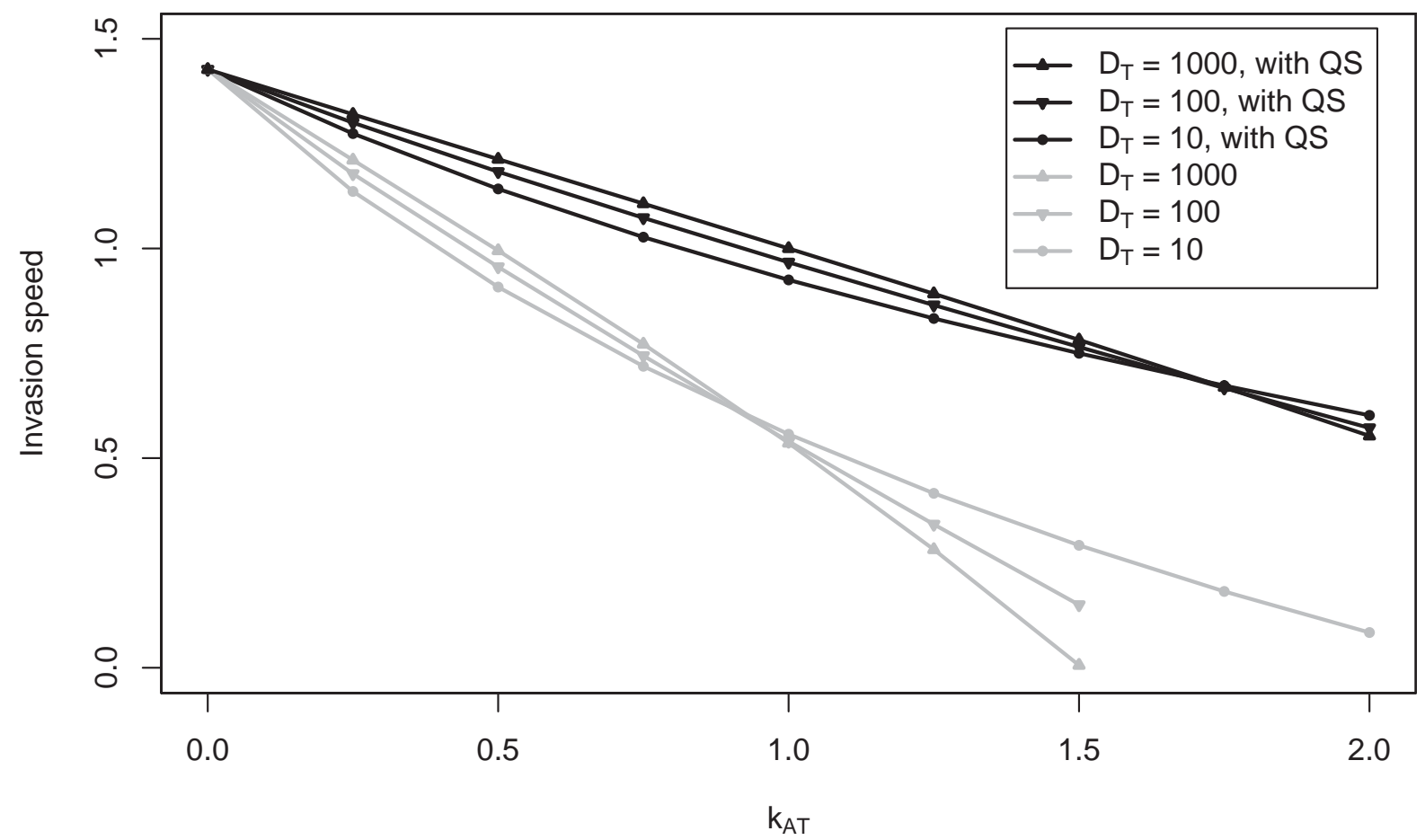

Figure 4: Invasion speeds when the established taxon produces a toxin regulated by quorum sensing. With quorum sensing (black), the level of toxin expression is, by design, half that specified in the case with constitutive toxin production (grey, curves are the same as in Fig. 2). Notice that this means changes in invasion speeds with $k_{A T}$ are less marked for QS regulated scenarios (black). Consequently, in the QS example, invasion speeds are closer to their maximum value, which corresponds to no defensive toxin production. Nevertheless, for both sets of curves we see the same qualtitative trends - at low $k_{A T}$ invasion speeds are slower when $D_{T}$ is low (e.g., $D_{T}=10$ ) but at high $k_{A T}$ invasion speeds are slower when $D_{T}$ is high (e.g., $D_{T}=1000$ ). Results are shown for $D_{R} / D_{T}=1$ and invasion speeds do not change significantly for $D_{R}<D_{T}$ or $D_{R}>D_{T}$. 


\section{DISCUSSION}

Many studies have examined the environmental factors that promote toxin production, but less is known about which types of toxins should evolve under different conditions. This is surprising given the large range of physical and chemical properties exhibited by the toxins secreted by microorganisms $32,38-41,43,47$. Our work paves the way for understanding how and why different toxin characteristics may be favored, depending on the various selection pressures faced by bacteria. For example, we find that fast toxin diffusion can be advantageous for an invader moving into a region that is already occupied by a competitor. However, when an established taxon is defending against an invader, slower toxin diffusion can be more effective, at least when the killing efficacy of the toxin is low (i.e., the strategy is to keep the weaker toxin at the invader wavefront where it is more effective at halting the invader, as opposed to the toxin diffusing to regions where it is less likely to have lasting impacts). This finding needs reconciling with real-world properties of toxins because large and more potent molecules generally diffuse slower than small and less potent molecules. This may represent a physical constraint; however, it is worth investigating whether and how different bacteria may have partially or fully overcome this constraint. Indeed, combining our approach with an explicit assessment of the costs involved in toxin production-related to toxin size, complexity and metabolic burden, among other considerations-would help explain the use of different toxins by microbes across different microbial niches.

Here we focused on modeling toxins that are not used up during the killing process. This is the simplest case and is appropriate for mechanisms where killing results from a change in ambient conditions, such as acidification of the environment by bacteria. This assumption also covers mechanisms where killing involves the action of a compound that can quickly revert back to its 
original state, such as enzymes based on redox chemistry. With other mechanisms, however, the toxin is destroyed or made otherwise unavailable during killing. This could occur, for example, if the toxin binds to cell walls or membranes in its target. Besides allowing for toxin consumption during use, another extension would be to consider the simultaneous production of different types of toxin. Many lactobacilli, for example, are capable of secreting a range of different compounds with different physical characteristics and modes of action ${ }^{60}$. It would be interesting to explore how interactions among different toxins might alter predictions of the current model. This also motivates the study of larger communities. Existing theoretical models of toxin production in three-member systems have shown that bacteriocins can promote diversity through non-transitive interactions ${ }^{54,55}$. It remains to be seen how similar patterns of toxin immunity and sensitivity might play out within the context of multiple concurrent invasions into established communities of diverse taxa. Nontransitive competition has also been observed among corals ${ }^{28}$, suggesting that an extended version of our approach would also be relevant to macroscopic systems involving allelopathy and allelochemical interactions. A final extension could involve invasion scenarios on planar surfaces (e.g., human mouths or gut walls), which could be tackled using a combination of the current approach and integro-difference methods ${ }^{7}$.

\section{CONCLUSION}

In this paper, we have taken a first step toward understanding how the non-local aspects of microbial competition alter classic predictions of invasion speeds. We found that toxin production can have a large effect on colonization success, with invasion speeds dependent on properties of the toxin, how toxin production is regulated and whether the toxin is used for offense or defense. Our work provides a basis for exploring further aspects of toxin use in the microbial world, including broader 
community analyses and investigation of evolutionary processes.

In conventional invasion models with competition ${ }^{1-4}$, a common analytical technique involves separating species into two teams such that each species cooperates with the other species in its team and competes with those in the other team. For our system (Eqn 1), one team is the invader and its toxin $(A$ and $S)$ while the other team is the established taxon and its toxin $(B$ and $T)$. This allows invasion speeds to be determined under the condition of linear determinacy ${ }^{3}$ by using a change of variables to convert the competition model into a cooperative system. However, linear determinacy conditions (e.g., Eqn 16) may not be satisfied if toxin diffusion is fast (i.e., $D_{S}, D_{T} \gg D_{A}, D_{B}$ ). Indeed, our analytical approximations for invasion speeds, which are supported by numerical simulations, show that invasion speeds are above those predicted by linear determinacy when $D_{S}$ and $D_{T}$ are large and $D_{A}$ and $D_{B}$ are nonzero. Therefore, our phenomenological approach to invasion speeds motivates the search for rigorous proofs for invasion speeds in systems with diffusible toxins. This will be a challenging but important line of inquiry because new analytical techniques are required to explore how nonlinear growth and interspecific interactions affect spatial spreading.

For many years, macroscopic ecological systems have inspired a wealth of mathematical systems with interesting biological and dynamical features. With advances in technology, we are now in a position to explore many of the same questions of microorganisms that have been posed for decades with larger organisms. And while many fundamental behaviors are retained across scales, microbes bring with them distinct traits and characteristics that are not found, or even possible, in macro-scale communities. Indeed, as demonstrated by our example of toxins and long-range competition, the microbial world poses new and interesting questions. Questions that give us the opportunity to learn more about the tools we know well and the systems we are only beginning to discover. 


\section{Linear determinacy conditions for the PDE system where two bacterial taxa produce toxins}

$$
\begin{aligned}
& \frac{\partial A}{\partial t}=D_{A} \frac{\partial^{2} A}{\partial x^{2}}+r_{A} A\left(1-\alpha_{A B}-A+\alpha_{A B} \widetilde{B}\right)-k_{A T} A\left(\frac{r_{T}}{m_{T}}-\widetilde{T}\right) \\
& \frac{\partial S}{\partial t}=D_{S} \frac{\partial^{2} S}{\partial x^{2}}+r_{S} A-m_{S} S \\
& \frac{\partial \widetilde{B}}{\partial t}=D_{B} \frac{\partial^{2} \widetilde{B}}{\partial x^{2}}+r_{B}(1-\widetilde{B})\left(\alpha_{B A} A-\widetilde{B}\right)+k_{B S}(1-\widetilde{B}) S \\
& \frac{\partial \widetilde{T}}{\partial t}=D_{T} \frac{\partial^{2} \widetilde{T}}{\partial x^{2}}+r_{T} \widetilde{B}-m_{T} \widetilde{T}
\end{aligned}
$$

which is cooperative in the biologically-realistic range $0 \leq A \leq 1 ; 0 \leq \widetilde{B} \leq 1 ; 0 \leq S \leq \frac{r_{S}}{m_{S}}$ and $0 \leq \widetilde{T} \leq \frac{r_{T}}{m_{T}}$. The moment generating matrix associated with the linearized version of Eqn 12 is

$$
\left[\begin{array}{cccc}
D_{A} \mu^{2}+r_{A}\left(1-\alpha_{A B}\right)-\frac{k_{A T} r_{T}}{m_{T}} & 0 & 0 & 0 \\
r_{S} & D_{S} \mu^{2}-m_{S} & 0 & 0 \\
r_{B} \alpha_{B A} & k_{B S} & D_{B} \mu^{2}-r_{B} & 0 \\
0 & 0 & r_{T} & D_{T} \mu^{2}-m_{T}
\end{array}\right]
$$

where $\mu$ is a variable. The first eigenvalue of the moment generating matrix is $\lambda_{1}(\mu)=D_{A} \mu^{2}+$ $r_{A}\left(1-\alpha_{A B}\right)-\frac{k_{A T} r_{T}}{m_{T}}$, which is associated with an invasion speed $c^{*}=\inf _{\mu>0} \frac{\lambda_{1}(\mu)}{\mu}=\frac{\lambda_{1}\left(\mu_{1}\right)}{\mu_{1}}=$ $2 \sqrt{D_{A} r_{A}\left(1-\alpha_{A B}-\frac{k_{A T} r_{T}}{r_{A} m_{T}}\right)}$ with $\mu_{1}=\sqrt{\frac{r_{A}\left(1-\alpha_{A B}-\frac{k_{A} r_{T}}{r_{A} m_{T}}\right)}{D_{A}}}$. The first eigenvalue at $\mu=\mu_{1}$ has a corresponding eigenvector: 


$$
\left[\begin{array}{l}
\zeta_{1} \\
\zeta_{2} \\
\zeta_{3} \\
\zeta_{4}
\end{array}\right]
$$

394

where

$$
\begin{aligned}
& \zeta_{1}=\frac{\left(\lambda_{1}\left(\mu_{1}\right)-D_{S} \mu_{1}^{2}+m_{S}\right)\left(\lambda_{1}\left(\mu_{1}\right)-D_{B} \mu_{1}^{2}+r_{B}\right)\left(\lambda_{1}\left(\mu_{1}\right)-D_{T} \mu_{1}^{2}+m_{T}\right)}{r_{S} k_{B S}+r_{B} \alpha_{B A}\left(\lambda_{1}\left(\mu_{1}\right)-D_{S} \mu_{1}^{2}+m_{S}\right)} \\
& \zeta_{2}=\frac{r_{S}\left(\lambda_{1}\left(\mu_{1}\right)-D_{B} \mu_{1}^{2}+r_{B}\right)\left(\lambda_{1}\left(\mu_{1}\right)-D_{T} \mu_{1}^{2}+m_{T}\right)}{r_{S} k_{B S}+r_{B} \alpha_{B A}\left(\lambda_{1}\left(\mu_{1}\right)-D_{S} \mu_{1}^{2}+m_{S}\right)} \\
& \zeta_{3}=\lambda_{1}\left(\mu_{1}\right)-D_{T} \mu_{1}^{2}+m_{T} \\
& \zeta_{4}=r_{T}
\end{aligned}
$$

Linear determinacy equates the invasion speed for the nonlinear system in Eqn 12 with the invasion speed in the linearized system, as long as Eqn 12 is dominated by the linearized system in the 397 direction determined by the above eigenvector ${ }^{3}$. Conditions for linear determinacy are then

$$
\begin{gathered}
\lambda_{1}\left(\mu_{1}\right)-D_{S} \mu_{1}^{2}+m_{S}>0 \\
\lambda_{1}\left(\mu_{1}\right)-D_{B} \mu_{1}^{2}+r_{B}>0 \\
\lambda_{1}\left(\mu_{1}\right)-D_{T} \mu_{1}^{2}+m_{T}>0 \\
\zeta_{3} \leq \min \left\{\frac{r_{A} \zeta_{1}-k_{A T} \zeta_{4}}{r_{A} \alpha_{A B}}, \frac{r_{B} \alpha_{B A} \zeta_{1}+k_{B S} \zeta_{2}}{r_{B}}\right\}
\end{gathered}
$$


398

406

407

5

which are relevant for Eqns 1,2 and 3. The conditions given in Eqn 16 ensure that the invasion speed of the nonlinear model (Eqn 12) is the above $c^{* 3}$.

\section{APPENDIX II}

\section{Analytical approximation for the toxin wavefront of the invading taxon}

Consider an invading taxon that is not strongly affected by the established taxon (or any toxin produced by the established taxon). This is equivalent to invasion in the absence of a competitor and

Eqn 1 reduces to

$$
\begin{aligned}
& \frac{\partial A}{\partial t}=D_{A} \frac{\partial^{2} A}{\partial x^{2}}+r_{A} A(1-A) \\
& \frac{\partial S}{\partial t}=D_{S} \frac{\partial^{2} S}{\partial x^{2}}+r_{S} A-m_{S} S
\end{aligned}
$$

We nondimensionalize Eqn 17 by making the following substitutions:

$$
\tau=r_{A} t
$$

$$
\rho=\frac{D_{S}}{D_{A}}
$$

$$
s=\frac{r_{A}}{r_{S}} S
$$

$$
\begin{array}{r}
\chi=x \sqrt{\frac{r_{A}}{D_{A}}} \\
\omega=\frac{m_{S}}{r_{A}} \\
a=A
\end{array}
$$

which gives

$$
\begin{aligned}
& \frac{\partial a}{\partial \tau}=\frac{\partial^{2} a}{\partial \chi^{2}}+a(1-a) \\
& \frac{\partial s}{\partial \tau}=\rho \frac{\partial^{2} s}{\partial \chi^{2}}+A-\omega s
\end{aligned}
$$


and therefore

$$
\begin{aligned}
& -c \frac{\partial a}{\partial z}=\frac{\partial^{2} a}{\partial z^{2}}+a(1-a) \\
& -c \frac{\partial s}{\partial z}=\rho \frac{\partial^{2} s}{\partial z^{2}}+A-\omega s
\end{aligned}
$$

As Eqn 20a does not depend on Eqn 20b, an approximate solution follows directly by using a standard approach for solving Fisher's equation. Substituting $Z=z / c$ in Eqn 20a gives

$$
-\frac{\partial a}{\partial Z}=\varepsilon \frac{\partial^{2} a}{\partial Z^{2}}+a(1-a)
$$

where $\varepsilon=1 / c^{2}$. Expanding $a$ in powers of $\varepsilon$ gives

$$
a(Z, \varepsilon)=a_{0}(Z)+\varepsilon a_{1}(Z)+\varepsilon^{2} a_{2}(Z) \ldots
$$

$$
-\frac{\partial a_{0}}{\partial Z}=a_{0}\left(1-a_{0}\right)
$$

$$
A \approx a_{0}=\frac{1}{1+e^{Z}}=\frac{1}{1+e^{z / c}}
$$




$$
-c \frac{\partial s}{\partial z}=\rho \frac{\partial^{2} s}{\partial z^{2}}+\frac{1}{1+e^{z / c}}-\omega s
$$

${ }_{417}$ As we are interested in the case where the toxin diffuses ahead of the invader wavefront, we assume ${ }_{418} \rho \gg 1$. Substituting $\rho=1 / \varrho^{2}$ in Eqn 25 we obtain

$$
-c \frac{\partial s}{\partial z}=\frac{1}{\varrho^{2}} \frac{\partial^{2} s}{\partial z^{2}}+\frac{1}{1+e^{z / c}}-\omega s
$$

419 where $\varrho \ll 1$. Following Olsen et $a l .{ }^{61}$, we can rescale $z$ such that the domain of influence of the 420 toxin is invariant in a new $\xi$-domain, where $\xi=\varrho z$. Eqn 26 then becomes

$$
-c \varrho \frac{\partial s}{\partial \xi}=\frac{\partial^{2} s}{\partial \xi^{2}}+\frac{1}{1+e^{\xi / \varrho c}}-\omega s
$$

As $\varrho \ll 1$, Eqn 27 can be approximated as

$$
\begin{array}{ll}
0 \approx \frac{\partial^{2} s}{\partial \xi^{2}}+1-\omega s & \text { for } \xi<0 \\
0 \approx \frac{\partial^{2} s}{\partial \xi^{2}}-\omega s & \text { for } \xi>0
\end{array}
$$

422

Solving Eqn 28 when assuming that $\lim _{\xi \rightarrow \infty} s(\xi)=0, \lim _{\xi \rightarrow-\infty} s(\xi)=1 / \omega$ and $s(0)=1 / 2 \omega$ gives

$$
\begin{array}{ll}
s(\xi) \approx-\frac{1}{2 \omega}\left(e^{\sqrt{\omega} \xi}-2\right) & \text { for } \xi<0 \\
s(\xi) \approx \frac{1}{2 \omega} e^{-\sqrt{\omega} \xi} & \text { for } \xi>0
\end{array}
$$

And rewriting Eqn 29 in terms of original parameters: 


$$
\begin{array}{ll}
S(z) \approx-\frac{r_{S}}{2 m_{S}}\left(e^{\sqrt{\frac{D_{A} m_{S}}{D_{S} r_{A}}} z}-2\right) & \text { for } z<0 \\
S(z) \approx \frac{r_{S}}{2 m_{S}} e^{-\sqrt{\frac{D_{A} m_{S}}{D_{S} r_{A}}} z} & \text { for } z>0
\end{array}
$$

\section{Analytical approximation for the toxin wavefront of the established taxon}

Consider a retreating population of established taxon due to invasion by a competitor taxon. For the special case that $\alpha_{B A}=2, r_{A}=r_{B}$ and $D_{A}=D_{B}$, and assuming that the invader does not produce a toxin and is not inhibited by the established population or its toxin, Eqn 1 reduces to

$$
\begin{aligned}
\frac{\partial A}{\partial t} & =D_{A} \frac{\partial^{2} A}{\partial x^{2}}+r_{A} A(1-A) \\
\frac{\partial B}{\partial t} & =D_{A} \frac{\partial^{2} B}{\partial x^{2}}+r_{A} B(1-B-2 A) \\
\frac{\partial T}{\partial t} & =D_{T} \frac{\partial^{2} T}{\partial x^{2}}+r_{T} B-m_{T} T
\end{aligned}
$$




$$
\tau=r_{A} t
$$

431

$$
\begin{gathered}
\rho=\frac{D_{T}}{D_{A}} \\
y=\frac{r_{A}}{r_{T}} T
\end{gathered}
$$

$$
\begin{array}{r}
\chi=x \sqrt{\frac{r_{A}}{D_{A}}} \\
\omega=\frac{m_{T}}{r_{A}} \\
a=A \\
b=B
\end{array}
$$

432 which gives

$$
\begin{aligned}
& \frac{\partial a}{\partial \tau}=\frac{\partial^{2} a}{\partial \chi^{2}}+a(1-a) \\
& \frac{\partial b}{\partial \tau}=\frac{\partial^{2} b}{\partial \chi^{2}}+b(1-b-2 a) \\
& \frac{\partial y}{\partial \tau}=\rho \frac{\partial^{2} y}{\partial \chi^{2}}+B-\omega y
\end{aligned}
$$

As above, we substitute $z=\chi-c \tau$ to shift to a moving reference frame, where now $c$ simultaneously represents the invasion speed, the retreating speed of the established population and the retreating speed of the toxin. This gives

$$
\begin{aligned}
& -c \frac{\partial a}{\partial z}=\frac{\partial^{2} a}{\partial z^{2}}+a(1-a) \\
& -c \frac{\partial b}{\partial z}=\frac{\partial^{2} b}{\partial z^{2}}+b(1-b-2 a) \\
& -c \frac{\partial y}{\partial z}=\rho \frac{\partial^{2} y}{\partial z^{2}}+B-\omega y
\end{aligned}
$$

\footnotetext{
Because we set $\alpha_{B A}=2$, notice that a solution to Eqns 34a and 34b is $b=1-a$. This allows us to approximate the wavefront of the retreating, established population as
} 


$$
B \approx b_{0}=1-\frac{1}{1+e^{z / c}}
$$

Solving Eqn 38 and rewriting in terms of original parameters:

$$
\begin{array}{ll}
T(z) \approx \frac{r_{T}}{2 m_{T}} e^{-\sqrt{\frac{D_{A} m_{T}}{D_{T} r_{A}}} z} & \text { for } z<0 \\
T(z) \approx-\frac{r_{T}}{2 m_{T}}\left(e^{\sqrt{\frac{D_{A} m_{T}}{D_{T} r_{A}}} z}-2\right) & \text { for } z>0
\end{array}
$$


which is the shape of the toxin wavefront produced by the established taxon in a moving reference frame, assuming that the invader does not produce a toxin and is not strongly inhibited by the established taxon or its toxin. Unsurprisingly, Eqn 39 is the mirror opposite of Eqn 30.

\section{APPENDIX III}

\section{Analytical approximations for invasion speeds when the invading taxon produces a toxin}

The conventional approach to calculating invasion speeds (without toxin) is to convert a competitive system like Eqn 2 to a linear cooperative system. This is done using the substitution $B=B^{\ddagger}-$ $\delta$, where $B^{\ddagger}$ is the population density of the established taxon before the arrival of the invading population and $\delta$ is a small perturbation. This approach, however, is not as straightforward when the invading taxon produces a toxin with non-local effects.

Consider Eqn 2a. Substituting $B=B^{\ddagger}-\delta$ and retaining only first order terms gives

$$
\frac{\partial A}{\partial t} \approx D_{A} \frac{\partial^{2} A}{\partial x^{2}}+r_{A} A\left(1-\alpha_{A B} B^{\ddagger}\right)
$$

In a moving reference frame with invasion speed $c$, i.e., $z=x-c t$, Eqn 40 becomes

$$
-c \frac{\partial A}{\partial z} \approx D_{A} \frac{\partial^{2} A}{\partial z^{2}}+r_{A} A\left(1-\alpha_{A B} B^{\ddagger}\right)
$$

Eqn 41 has exponential solutions $A=f(z)=e^{-\mu z}$ where $c \mu=D_{A} \mu^{2}+r_{A}\left(1-\alpha_{A B} B^{\ddagger}\right)$. Solving for $\mu$ : 


$$
\mu=\frac{c \pm\left(c^{2}-4 D_{A} r_{A}\left(1-\alpha_{A B} B^{\ddagger}\right)\right)^{\frac{1}{2}}}{2 D_{A}}
$$

which is real if $c>c^{*}=2 \sqrt{D_{A} r_{A}\left(1-\alpha_{A B} B^{\ddagger}\right)}$. However, if $B^{\ddagger}=1$ then it is clear that we have linearized away the effect of the toxin. We therefore require a modified value for $B^{\ddagger}$ which takes into account the negative effect of the toxin on the established taxon.

If we assume that the toxin is fast diffusing then $r_{B} \alpha_{B A} A \ll k_{B S} S$. With this assumption, Eqn 2c can be approximated as

$$
\frac{\partial B}{\partial t} \approx D_{B} \frac{\partial^{2} B}{\partial x^{2}}+r_{B} B(1-B)-k_{B S} B S
$$

If we assume that the killing rate of the toxin is large then $k_{B S} \gg 1$ and $D_{B} / k_{B S} \ll 1$; if we also assume that the growth rate of the established taxon is sufficiently fast to keep pace with killing due to the toxin then $r_{B} / k_{B S} \sim 1$. With these assumptions, Eqn 43 simplifies to

$$
B=1-\frac{\kappa}{\sigma} S=1-\frac{k_{B S}}{r_{B}} S
$$

which is an expression for the density of the established taxon for a given density of toxin. Let us assume that toxin density can be described by Eqn 30, even though this expression is only strictly correct in the case that the established taxon does not inhibit the invader. Clearly, $S=0$ when $D_{S}=0$, which suggests that in the limit $D_{S} \rightarrow 0$ the system exhibits only local competition. For this case, it is well known that the competitive strength of the invader has no influence on invasion 
speed $^{14}$, which is then given by the classic result:

$$
c_{0}^{*}=2 \sqrt{D_{A} r_{A}\left(1-\alpha_{A B}\right)}
$$

If, however, the toxin diffuses ahead of the invader wavefront, then we can assume that the population density of the established taxon is reduced by an amount commensurate with toxin density, which, in the limit of infinitely-fast toxin diffusion, is given by Eqn 30 with $D_{S}=\infty$, i.e.,

$$
B_{\infty}^{\ddagger}=1-\frac{k_{B S} r_{S}}{2 m_{S} r_{B}}
$$

For real solutions to $\mu$ in Eqn 42 with $B_{\infty}^{\ddagger}$ :

$$
\begin{array}{ll}
c_{\infty}^{*}=2 \sqrt{D_{A} r_{A}\left(1-\alpha_{A B}\left(1-\frac{k_{B S} r_{S}}{2 m_{S} r_{B}}\right)\right)} & \text { for } k_{B S} r_{S}<2 m_{S} r_{B} \\
c_{\infty}^{*}=2 \sqrt{D_{A} r_{A}} & \text { for } k_{B S} r_{S}>2 m_{S} r_{B}
\end{array}
$$

where we have set $B^{\ddagger}=0$ for situations where the toxin kills sufficiently quickly that the established taxon is eradicated across the entire domain.

\section{Analytical approximations for invasion speeds when the established taxon produces a toxin}

Consider Eqn 3. As in the previous section, we expand around $B=B^{\ddagger}-\delta$, where $B^{\ddagger}$ is the population density of the established taxon in the absence of the invader and $\delta$ is a small perturbation.

Retaining only first order terms in Eqn 3a gives

$$
\frac{\partial A}{\partial t} \approx D_{A} \frac{\partial^{2} A}{\partial x^{2}}+r_{A} A\left(1-\alpha_{A B} B^{\ddagger}\right)-k_{A T} A T
$$


482

483

484 485

486 487 488

The invasion speed in the limit of infinitely-fast toxin diffusion is then

$$
c_{\infty}^{*}=2 \sqrt{D_{A} r_{A}\left(1-\alpha_{A B}-\frac{k_{A T} r_{T}}{2 r_{A} m_{T}}\right)}
$$
becomes

$$
\frac{\partial A}{\partial t} \approx D_{A} \frac{\partial^{2} A}{\partial x^{2}}+r_{A} A\left(1-\alpha_{A B} B^{\ddagger}\right)-k_{A T} A T^{\ddagger}
$$
invasion speed in the limit of no diffusion of toxin:

$$
c_{0}^{*}=2 \sqrt{D_{A} r_{A}\left(1-\alpha_{A B}-\frac{k_{A T} r_{T}}{r_{A} m_{T}}\right)}
$$

$$
\frac{\partial A}{\partial t} \approx D_{A} \frac{\partial^{2} A}{\partial x^{2}}+r_{A} A\left(1-\alpha_{A B} B^{\ddagger}\right)-k_{A T} A \frac{r_{T}}{2 m_{T}}
$$

where we have set $B^{\ddagger}=1$.

In the limit of slow toxin diffusion $D_{T} \rightarrow 0$, we similarly assume that $T=T^{\ddagger}-\delta$ and Eqn 48

Eqn 49 has exponential solutions $A=f(z)=e^{-\mu z}$, which can be solved, as above, to give the 


\section{References}

1. Kot, M., Lewis, M.A. \& van den Driessche, P. Dispersal data and the spread of invading organisms. Ecology 77, 2027-2042 (1996).

2. Hastings, A. Models of spatial spread: is the theory complete? Ecology 77, 1675-1679 (1996).

3. Weinberger, H.F., Lewis, M.A. \& Li, B. Analysis of linear determinacy for spread in cooperative models. Journal of Mathematical Biology 45, 183-218 (2002).

4. Hastings, A. et al. The spatial spread of invasions: new developments in theory and evidence. Ecology Letters 8, 91-101 (2005).

5. Melbourne, B.A. et al. Invasion in a heterogeneous world: resistance, coexistence or hostile takeover? Ecology Letters 10, 77-94 (2007).

6. Li, B., Bewick, S., Shang, J. \& Fagan, W.F. Persistence and spread of a species with a shifting habitat edge. SIAM Journal on Applied Mathematics 74, 1397-1417 (2014).

7. Li, B., Bewick, S., Barnard, M.R. \& Fagan, W.F. Persistence and Spreading Speeds of IntegroDifference Equations with an Expanding or Contracting Habitat. Bulletin of Mathematical Biology $\mathbf{0}, 0$ (2016).

8. Skellam, J.G. Random dispersal in theoretical populations. Biometrika 38, 196-218 (1951).

9. Neubert, M.G. \& Caswell, H. Demography and dispersal: calculation and sensitivity analysis of invasion speed for structured populations. Ecology 81, 1613-1628 (2000).

10. Lewis, M.A. \& Kareiva, P. Allee dynamics and the spread of invading organisms. Theoretical Population Biology 43, 141-158 (1993).

11. Skalski, G.T. \& Gilliam, J.F. Modeling diffusive spread in a heterogeneous population: a movement study with stream fish. Ecology 81, 1685-1700 (2000).

12. Perkins, T.A., Phillips, B.L., Baskett, M.L. \& Hastings, A. Evolution of dispersal and life history interact to drive accelerating spread of an invasive species. Ecology Letters 16, 10791087 (2013). 
13. Shigesada, N., Kawasaki, K. \& Teramoto, E. Traveling periodic waves in heterogeneous environments. Theoretical Population Biology 30, 143-160 (1986).

14. Okubo, A., Maini, P.K., Williamson, M.H. \& J.D., Murray. On the spatial spread of the grey squirrel in britain. Proceedings of the Royal Society of London B: Biological Sciences 238, 113-125 (1989).

15. Owen, M.R. \& Lewis, M.A. How predation can slow, stop or reverse a prey invasion. Bulletin of Mathematical Biology 63, 655-684 (2001).

16. Van der Waaij, D., Berghuis-de Vries, J.M. \& Lekkerkerk-Van der Wees, J.E.C. Colonization resistance of the digestive tract in conventional and antibiotic-treated mice. Journal of Hygiene 69, 405-411 (1971).

17. Vollaard, E.J. \& Clasener, H.A. Colonization resistance. Antimicrobial Agents and Chemotherapy 38, 409-414 (1994).

18. Kessenich, C.R. \& Bah, A. Necrotizing Fasciitis: Understanding the deadly results of the uncommon 'flesh-eating bacteria'. The American Journal of Nursing 104, 51-55 (2004).

19. Wilson, M. \& Lindow, S.E. Coexistence among epiphytic bacterial populations mediated through nutritional resource partitioning. Applied and Environmental Microbiology 60, 44684477 (1994).

20. Hibbing, M.E., Fuqua, C., Parsek, M.R. \& Peterson, S.B. Bacterial competition: surviving and thriving in the microbial jungle. Nature Reviews Microbiology 8, 15-25 (2010).

21. Bibel, D.J. et al. Competitive adherence as a mechanism of bacterial interference. Canadian Journal of Microbiology 29, 700-703 (1983).

22. Miller, M.B. \& Bassler, B.L. Quorum sensing in bacteria. Annual Reviews in Microbiology 55, 165-199 (2001).

23. Waters, C.M. \& Bassler, B.L. Quorum sensing: cell-to-cell communication in bacteria. Annual Review of Cell and Developmental Biology 21, 319-346 (2005). 
24. Müller, J., Kuttler, C., Hense, B.A., Rothballer, M. \& Hartmann, A. Cell-cell communication by quorum sensing and dimension-reduction. Journal of Mathematical Biology 53, 672-702 (2006).

25. Hense, B.A. et al. Does efficiency sensing unify diffusion and quorum sensing? Nature Reviews Microbiology 5, 230-239 (2007).

26. Frederick, M.R., Kuttler, C., Hense, B.A. \& Eberl, H.J. A mathematical model of quorum sensing regulated EPS production in biofilm communities. Theoretical Biology and Medical Modelling 8, 1-29 (2011).

27. Jackson, J.B.C. \& Buss, L.W. Allelopathy and spatial competition among coral reef invertebrates. Proceedings of the National Academy of Sciences USA 72, 5160-5163 (1975).

28. Buss, L.W. \& Jackson, J.B.C. Competitive networks: Nontransitive competitive relationships in cryptic coral reef environments. American Naturalist 113, 223-234 (1979).

29. Lang, J.C. \& Chornesky, E.A. Competition between scleractinian reef corals-A review of mechanisms and effects. Ecosystems of the World 25, 209-252 (1990).

30. Chadwick, N.E. \& Morrow, K.M. Competition among sessile organisms on coral reefs. In Coral Reefs: An Ecosystem in Transition, 347-371 (Springer Netherlands, 2011).

31. Shu, M. et al. Fermentation of Propionibacterium acnes, a commensal bacterium in the human skin microbiome, as skin probiotics against methicillin-resistant Staphylococcus aureus. PLoS One 8, e55380 (2013).

32. Boris, S. \& Barbés, C. Role played by lactobacilli in controlling the population of vaginal pathogens. Microbes and Infection 2, 543-546 (2000).

33. A.N., Skarin \& Sylwan, J.E. Vaginal lactobacilli inhibiting growth of Gardnerella vaginalis, Mobiluncus and other bacterial species cultured from vaginal content of women with bacterial vaginosis. Acta Pathologica Microbiologica Scandinavica Series B: Microbiology 94, 399-403 (1986). 
34. Dahiya, R.S. \& Speck, M.L. Hydrogen peroxide formation by lactobacilli and its effect on Staphylococcus aureus. Journal of Dairy Science 51, 1568-1572 (1968).

35. Pericone, C.D., Overweg, K., Hermans, P.W. \& Weiser, J.N. Inhibitory and bactericidal effects of hydrogen peroxide production by Streptococcus pneumoniae on other inhabitants of the upper respiratory tract. Infection and immunity 68, 3990-3997 (2000).

36. Riley, M.A. \& Wertz, J.E. Bacteriocins: evolution, ecology, and application. Annual Reviews in Microbiology 56, 117-137 (2002).

37. Ramu, R. et al. Bacteriocins and their applications in food preservation. Critical Reviews in Food Science and Nutrition 0, 0 (2015).

38. Balciunas, E.M. et al. Novel biotechnological applications of bacteriocins: A review. Food Control 32, 134-142 (2013).

39. Cotter, P.D., Ross, R.P. \& Hill, C. Bacteriocins-a viable alternative to antibiotics? Nature Reviews Microbiology 11, 95-105 (2013).

40. Cavera, V.L., Arthur, T.D., Kashtanov, D. \& Chikindas, M.L. Bacteriocins and their position in the next wave of conventional antibiotics. International Journal of Antimicrobial Agents 46, 494-501 (2015).

41. Moll, G.N., Konings, W.N. \& Driessen, A.J.M. Bacteriocins: mechanism of membrane insertion and pore formation. In Lactic acid bacteria: Genetics, metabolism and applications, 185-198 (Springer Netherlands, 1999).

42. Mora, L. \& de Zamaroczy, M. In vivo processing of DNase colicins E2 and E7 is required for their import into the cytoplasm of target cells. PLoS One 19, e96549 (2014).

43. $\mathrm{Ng}$, C.L. et al. Structural basis for $16 \mathrm{~S}$ ribosomal RNA cleavage by the cytotoxic domain of colicin E3. Nature Structural and Molecular Biology 17, 1241-1246 (2010).

44. Cotter, P.D., Hill, C. \& Ross, R.P. Bacteriocins: developing innate immunity for food. Nature Reviews Microbiology 3, 777-788 (2005). 
45. Kanagaraj, J., Senthivelan, T., Panda, R.C. \& Kavitha, S. Eco-friendly waste management strategies for greener environment towards sustainable development in leather industry: a comprehensive review. Journal of Cleaner Production 89, 1-17 (2015).

46. Yang, S.-C., Lin, C.-H., Sung, C.T. \& Fang, J.-Y. Antibacterial activities of bacteriocins: application in foods and pharmaceuticals. Frontiers in Microbiology 5, 241 (2014).

47. Zheng, J., Gänzle, M.G., Lin, X.B., Ruan, L. \& Sun, M. Diversity and dynamics of bacteriocins from human microbiome. Environmental Microbiology 17, 2133-2143 (2015).

48. Gillor, O., Etzion, A. \& Riley, M.A. The dual role of bacteriocins as anti- and probiotics. Applied Microbiology and Biotechnology 81, 591-606 (2008).

49. Frank, S.A. Spatial polymorphism of bacteriocins and other allelopathic traits. Evolutionary Ecology 8, 369-386 (1994).

50. Durrett, R. \& Levin, S. Allelopathy in spatially distributed populations. Journal of Theoretical Biology 185, 165-171 (1997).

51. Kerr, B. The ecological and evolutionary dynamics of model bacteriocin communities. In Bacteriocins, 111-134 (Springer Berlin Heidelberg, 2007).

52. Gordon, D.M. \& Riley, M.A. A theoretical and empirical investigation of the invasion dynamics of colicinogeny. Microbiology 145, 655-661 (1999).

53. Nakamaru, M. \& Iwasa, Y. Competition by allelopathy proceeds in traveling waves: colicinimmune strain aids colicin-sensitive strain. Theoretical Population Biology 57, 131-144 (2000).

54. Lenski, R.E. \& Riley, M.A. Chemical warfare from an ecological perspective. Proceedings of the National Academy of Sciences USA 99, 556-558 (2002).

55. Czárán, T.L., Hoekstra, R.F. \& Pagie, L. Chemical warfare between microbes promotes biodiversity. Proceedings of the National Academy of Sciences USA 99, 786-790 (2002).

56. Blanchard, A.E., Liao, C. \& Lu, T. An ecological understanding of quorum sensing-controlled bacteriocin synthesis. Cellular and Molecular Bioengineering 0, 0 (2016). 
57. Gardner, A., West, S.A. \& Buckling, A. Bacteriocins, spite and virulence. Proceedings of the Royal Society of London B: Biological Sciences 271, 1529-1535 (2004).

58. Czárán, T. \& Hoekstra, R.F. A spatial model of the evolution of quorum sensing regulating bacteriocin production. Behavioral Ecology 18, 866-873 (2007).

59. Fisher, R.A. The wave of advance of advantageous genes. Annals of Eugenics 7, 355-369 (1937).

60. Schillinger, U. Bacteriocins of lactic acid bacteria. In Biotechnology and Food Safety: Proceedings of the Second International Symposium, 55-74 (Elsevier, 2014).

61. Olsen, L., Sherratt, J.A., Maini, P.K. \& Arnold, F. A mathematical model for the capillary endothelial cell-extracellular matrix interactions in wound-healing angiogenesis. Mathematical Medicine and Biology 14, 261-281 (1997).

Acknowledgements We thank one anonymous reviewer for comments. This work was supported in part by the U.S. Army Research Laboratory and the U.S. Army Research Office under contract/grant number \#W911NF-14-1-0490 (DK). Additional support came from the National Science Foundation under Grant DMS-1225917 (WFF) and Grants DMS-1225693 and DMS-1515875 (BL).

Competing Interests The authors declare that they have no competing financial interests.

Contributions All authors discussed results and edited the manuscript.

Correspondence Correspondence should be addressed to sharon_bewick@ hotmail.com 